\title{
Oxidative and Antioxidative Status after Successful Percutaneous Transluminal Coronary Angioplasty in Acute Myocardial Infarction
}

Hakan Tas M$^{1 *}$, Mehmet Emin Kalkan², Enbiya Aksakal ${ }^{1}$, Ziya Simsek ${ }^{1}$, Eftal Murat Bakirci ${ }^{1}$ and Hamit Hakan Alp $^{3}$

${ }^{1}$ Department of Cardiology, Faculty of Medicine, Ataturk University, Turkey

${ }^{2}$ Kartal Kosuyolu Yuksek Ihtisas Heart Education and Research Hospital, Department of Cardiology, Turkey

${ }^{3}$ Department of Biochemistry, Ataturk University, Turkey

Received: December 02, 2013; Accepted: February 13, 2014, Published: February 14, 2014

*Corresponding author: Hakan Tas M, Ataturk University, Cardiology Department, 25240-Erzurum, Turkey, Tel: +90-532-685-6848; Fax: +90-442235-2384; E-mail: mhakantas@gmail.com

\begin{abstract}
Background: Oxidants lead to cell death through apoptosis and necrosis. The effects of reactive oxygen radicals are balanced by the antioxidant action. The aim of this study was to assess and compare the oxidative and antioxidative status, both in healthy control subjects and in patients with acute myocardial infarction after successful Percutaneous Transluminal Coronary Angioplasty (PTCA).
\end{abstract}

Materials and methods: 25 patients who were re-vascularised in the first six hours of Acute ST elevated myocardial infarction and 25 healthy controls were included in the study. Erythrocyte Total Superoxide Scavenger Activity (TSSA), Non-enzymatic Superoxide Scavenger Activity (NSSA), superoxide dismutase (SOD), Nitric Oxide (NO), glutathione peroxidase (GPX) activities and glutathione (GSH), malondialdehyde (MDA) and 8 hydroxydeoxyguanosine (8-OHdG) levels were measured before PTCA (zero-time) after the $6^{\text {th }}$ hour and $24^{\text {th }}$ hour from PTCA in patients and once in the controls.

Results: There was a decrease at MDA concentrations $(3.99 \pm 1.14$, $4.05 \pm 1.35, \quad 3.62 \pm 1.11 \mathrm{nmol} / \mathrm{g} \mathrm{Hb}, \mathrm{p}<0.05$ ), however, 8-OHdG concentrations significantly increased in patient at zero-time and $6^{\text {th }}$ hours after PTCA $\left(2.52 \pm 1.27,2.56 \pm 1.3610^{6} \mathrm{dG}, \mathrm{p}<0.001\right)$ and a lower significant value at the $24^{\text {th }}$ hour $\left(2.50 \pm 1.6310^{6} \mathrm{dG}, \mathrm{p}=0.001\right)$. TSSA, NSSA, SOD, NO, GPX, GSH significantly increased in patients in zerotime, $6^{\text {th }}$ hour and $24^{\text {th }}$ hour of acute myocardial infarction. After PTCA an increase of concentrations continued.

Conclusion: Prior studies reported an imbalance in the favour of oxidative stress in acute myocardial infarction after reperfusion. This study showed that PTCA and routine medical therapy downgrades the imbalance of oxidative and Anti oxidative status in favour of the Anti oxidative effect.

Keywords: Acute myocardial infarction; Oxidative stress; Antioxidant defence; Percutaneous transluminal coronary angioplasty

\section{Introduction}

Today, the prevalence of cardiovascular diseases has been increasing worldwide. Coronary artery disease constitutes the majority of these cardiovascular diseases. The rupture of luminal plaques, which consist of cholesterol and lipids, leads to the release of materials stimulating platelet activation and thrombin formation. As a result, a thrombus limiting blood flow is formed. This situation leads to an imbalance between oxygen demand and supply, and if it becomes permanent, it leads to acute myocardial infarction [1]. Previous studies have shown that the imbalance between formation of reactive oxygen species and antioxidant defence together with conventional risk factors play an important role in the pathogenesis of atherosclerosis. The production of reactive oxygen species start during ischemia and go on even after reperfusion. The excessive production of reactive oxygen species plays a role in the pathogenesis of atherosclerosis leading to oxidation of low density lipoproteins, endothelial dysfunction, growth of vascular smooth muscle and monocyte migration [2]. Percutaneous Transluminal Coronary Angioplasty (PTCA) is the leading method among mechanical revascularisation procedures. Reperfusion after ischemia can increase the production of oxidants, which can overcome antioxidant capacity. Some previous studies showed that an increase in the production of oxidants with a decrease in the production of antioxidants could cause an imbalance in favour of oxidants. Increase of oxidants, leading lipid peroxidation in cell membrane, damage of membrane proteins and, DNA fragmentation with myocardial cell injury, causes loss of contractile function. In some in vitro studies, a burst-like increase in free oxygen radicals after ischemia and reperfusion in an isolated heart was reported. Bolli R, et al. [3] found that free oxygen radicals increased in significant amounts in coronary venous blood after 15 minutes of coronary occlusion and reperfusion [3]. These studies reveal that the production of free oxygen radicals continues and increases significantly during reperfusion after ischemia. Studies on oxidative stress during PTCA have produced controversial results [4]. In our study we aimed to show oxidative and antioxidative status in the early phase of acute myocardial infarction and after PTCA.

\section{Materials and Method}

The study was performed in the Ataturk University Cardiology Department according to the criteria of the Helsinki Declaration. 
The study protocol was confirmed by the Ataturk University Medical Faculty Ethics Committee. Informed and signed consent was obtained from all patients.

Patients with Acute Myocardial Infarction (AMI) admitted to our clinic were included in the study. Inclusion criteria were AMI symptoms for more than 30 minutes, admission six hours after onset of symptoms, $>0.1 \mathrm{mv}$ ST segment elevation in two or more contiguous ECG leads, TIMI 0 flow with a total occlusion in coronary angiography and provision of TIMI 3 flow after PTCA. Exclusion criteria were lack of patient's consent for study, chronic renal failure and any chronic inflammatory disease. 25 patients with Acute Myocardial Infarction (AMI) treated successfully with PTCA and 25 healthy volunteers were included in the study.

Medical therapy in patients with the diagnosis of AMI was performed in accordance with the American College of Cardiology/American Heart Association guidelines. All patients were given $300 \mathrm{mg}$ acetylsalicylic acid (ASA) and $5000 \mathrm{IU}$ heparin before PTCA. A weight $(\mathrm{kg})$ adjusted tirofiban bolus was given to patients with thrombosed lesions during PTCA and the drug was continued at a maintenance dose after PTCA. In light of the guidelines, acethylsalisilic acid, clopidogrel, beta blockers (metoprolol, nebivolol), antihyperlipidemic agents and angiotensin converting enzyme inhibitors were added to therapy. Blood samples were collected into vacuum tubes with EDTA at admission ( 0 hour), sixth and $24^{\text {th }}$ hour. Only one blood sample was taken from the control group.

\section{Biochemical measurements}

Erythrocytes were sedimented by the centrifuge method and haemolysed with deionised water. The haemoglobin values of patients and the control group were determined by Gen-S haematology analysis device. The samples were kept at $-80^{\circ} \mathrm{C}$ until biochemical analyses were performed (up to 2 weeks). Total Superoxide Scavenger Activity (TSSA) and Non-enzymatic Superoxide Scavenger Activity (NSSA) measurements were performed before and after the addition of trichloroacetic acid (TCA, 20\%) into samples. TSSA measurement was performed first, then NSSA measurement was conducted with the removal of proteins by centrifugation of the sample added TCA $20 \%$ solution, at $5000 * \mathrm{~g}$ for 30 minutes. Superoxide dismutase (SOD) activity was calculated by subtracting NSSA from TSSA. The measurement of malonyldialdehyde (MDA), which is an important marker of oxidative stress, is achieved by spectrophotometric analysis of thiobarbituric acid reactive products (TBARS). Glutathione (GSH) is detected with the Beutler method [5]. The glutathione peroxidase activity was determined spectrophotometrically [6] The 8-hydroxydeoxiguanosine (8-OHdg) levels were measured by ELISA.

\section{Statistical analysis}

Data was analysed with SPSS 20.0 (Statistical Package for Social Sciences, SPSS Inc., Chicago, IL, USA). A Paired-Samples $\mathrm{T}$ test was used to compare parametric variables between the patient and control groups and Chi-square test for categorical variables. The Kolmogorov-Smirnov test was used to determine whether the variables were normally distributed. The continuous variables were expressed as mean \pm standard deviation categorical data was expressed as percent (\%). A p value $<0.05$ was considered to be statistically significant.

\section{Results}

Eighty-eight percent of 25 patients and $84 \%$ of 25 healthy volunteers were males. The mean age among both groups was similar $(55 \pm 5,52 \pm 6$; NS). Smokers constituted $48 \%$ of the patient group and $43 \%$ of the control group and there were no significant difference. Diabetes mellitus was found to be $4 \%$ in patient groups. Hypertension was detected in $16 \%$ of patients. In healthy volunteers there were no hypertension or diabetes mellitus patients. Anterior and inferior myocardial infarction constituted $52 \%$ and $48 \%$ of patients, respectively. The patients total cholesterol, high density lipoprotein and low density lipoprotein levels were significantly higher than the control group. All of the patients were given asethylsalisilic acid, clopidogrel, enoxaparine and statins. Beta-blockers and ACE inhibitors were used if required in $66 \%$ and $38 \%$ of patients, respectively, due to their rhythm and blood pressure values. Nitrates could be used in $23 \%$ of patients. Tirofiban was given to $95 \%$ of patients and started during PTCA with a bolus dose and a maintenance infusion was continued for 24 hours. The health status, general characteristics of subjects included in the study and the therapy given for patients are shown in Table 1 .

No significant increase was observed in malonylaldehyde levels between patients $(3.99 \pm 1.14,4.05 \pm 1.35,3.62 \pm 1.11 \mathrm{nmol} / \mathrm{g}$ $\mathrm{Hb}, \mathrm{p}<0.05)$ and the control group $(4.76 \pm 1.4 \mathrm{nmol} / \mathrm{g} \mathrm{Hb})$ at 0 , 6 th and 24th hours. 8-OHdG levels increased in higher significance at 0 and $6^{\text {th }}$ hours compared to the control group $(2.52 \pm 1.27$,

Table 1: The health status, general characteristics of subjects included in the study and the therapy given for patients.

\begin{tabular}{|c|c|c|c|}
\hline & $\begin{array}{l}\text { AMI group } \\
(n=25)\end{array}$ & $\begin{array}{l}\text { Control group } \\
(n=25)\end{array}$ & $P$ value \\
\hline Age & $55 \pm 5$ & $52 \pm 6$ & 0.41 \\
\hline Male (\%) & 88 & 84 & 0.27 \\
\hline Hypertension (\%) & 16 & - & - \\
\hline DiabetesMellitus (\%) & 4 & - & - \\
\hline CurrentSmoker (\%) & 48 & 43 & 0.07 \\
\hline PriorInfarction (\%) & 0 & 0 & - \\
\hline Total Cholesterol (mg\%) & $205 \pm 45$ & $154 \pm 39$ & $<0.001$ \\
\hline LDL cholesterol (mg/dl) & $150 \pm 28$ & $126 \pm 43$ & $<0.05$ \\
\hline HDL cholesterol (mg/dl) & $32 \pm 10$ & $41 \pm 12$ & $<0.001$ \\
\hline Aspirin (\%) & 100 & - & - \\
\hline ACE (\%) & 38 & - & - \\
\hline$\beta$-blocker (\%) & 66 & - & - \\
\hline Clopidogrel (\%) & 100 & - & - \\
\hline Tirofiban (\%) & 95 & - & - \\
\hline Enoxiparine (\%) & 100 & - & - \\
\hline Statin (\%) & 100 & - & - \\
\hline Nitrate (\%) & 23 & - & - \\
\hline
\end{tabular}


$\left.2.56 \pm 1.3610^{6} \mathrm{dG}, \mathrm{p}<0.001\right)$ and there was a significant increase at the $24^{\text {th }}$ hour $\left(2.50 \pm 1.6310^{6} \mathrm{dG}, \mathrm{p}=0.001\right)$. The levels of antioxidants like NSSA, TSSA, NO, SOD were significantly higher in the patient group than the control group at $0,6^{\text {th }}$ and $24^{\text {th }}$ hours. Compared to the control group, GPX and GSH levels were found to be higher in the patient group without higher statistical significance. There was no significant difference in levels of oxidants and antioxidants between 0 and $6^{\text {th }}, 0$ and $24^{\text {th }}$ and $6^{\text {th }}$ and $24^{\text {th }}$ hours. The concentrations of oxidants and antioxidants at $0,6^{\text {th }}$ and $24^{\text {th }}$ hours are shown in Table 2 .

\section{Discussion}

Myocardial ischemia can be detected in many conditions like atherosclerosis, thromboembolism, coronary artery bypass graft operation and percutaneous transluminal angioplasty. The common problem in all of these conditions is the inability to supply oxygen and substrates necessary for myocardial metabolism. Free oxygen radicals are formed by reduction of oxygen and under normal conditions they are removed by the body's scavenger systems. During myocardial ischemia there is an excessive production of these free oxygen radicals and the oxidative-antioxidative balance is impaired in favour of oxidation [7]. Our study partially supports the findings in previous studies related to oxidative and antioxidative state in patients with acute myocardial infarction. However, it provides some new data about the situation after PTCA. MDA is a ketoaldehyde found in great amounts after lipid peroxidation, assumed to be a marker of oxidative injury because of its relationship with lipoproteins. Cavalca V, et al. [8] detected a significant increase in MDA levels in patients with coronary artery disease compared to the control group. Pucheu S, et al. [9] reported that MDA is a useful marker in evaluation of oxidative stress after reperfusion of ischemic myocardium and they detected a significant increase in MDA levels in patients treated by intravenous thrombolytics compared to those without this therapy. In our study we found that there was no significant increase in MDA levels compared to the control group. However, a significant decrease was found in the patient group. This situation can be due to the effects of statin therapy started at admission and because of its elimination from the system after coronary reperfusion provided by PTCA 8-OHdG levels give us information about oxidative DNA damage and the activity of DNA repair mechanisms. The removal of lactic acid produced by myocardial metabolism decreases after ischemia, resulting in acidosis. The activity of DNA repair enzymes decreases as a result of acidosis. Reperfusion achieved by PTCA causes an increase in the activity of DNA repair enzymes. In rat studies 8-hydroxydeoxyguanosine levels were found to increase in infracted regions [10]. Nagayoshi Y, et al. [11] showed that in patients with acute myocardial infarction, 8-OHdG levels were high at the beginning, reached their peak at the fourth hour after reperfusion and started to decrease at the $24^{\text {th }}$ hour. In a study by Solen H, et al. [12] it was reported that in patients with acute myocardial infarction, the 8-OHdG levels were high in the first day and showed a sharp decline at the third day in patients treated by PTCA. Toth-Zsamboski E, et al. [13] found that 8-OHdG increased rapidly after PTCA and returned to normal levels after 96 hours. In our study, we detected that at the beginning and sixth hour after PTCA, 8-OHdG levels were significantly higher in the patient group than the control subjects, however, at the $24^{\text {th }}$ hour, 8-OHdG levels were higher again without higher statistical significance and started to decrease. These results are similar to previous studies and 8-OHdG levels can provide information about the success of PTCA in the early phase, although further studies are needed. This early cleaning from blood could be a cause of tirofiban usage.

Reperfusion therapy must be conducted as earlier as possible after myocardial infarction in order to prevent ischemic injury. However, reperfusion is known to cause additional myocardial damage. A hypothesis proposes that sudden increase in oxygen metabolism after reperfusion causes a kind of insufficiency of anti-oxidation during myocardial infarction in patients with deficient defence systems [14]. Nevertheless, unlike some previous studies and hypothesis, in our study we found that antioxidant levels did not decrease, furthermore some markers increased significantly. Dusinovic S, et al. [15] did not find any significant change in the activity of SOD and GPX at the beginning and 1, 3, 6 and 24 hours of thrombolytic therapy in AMI patients. Simic D, et al. [16] reported that in patients with acute myocardial infarction after successful reperfusion, a significant increase was detected in SOD and GPX activities at the first hour, which continued up to the third day. Tomado $\mathrm{H}$, et al. [17] showed that in AMI patients admitted 6 hours after symptom onset who had undergone PTCA, SOD activity started to increase at the first hour

Table 2: The concentrations of oxidants and antioxidants at $0,6^{\text {th }}$ and $24^{\text {th }}$ hour in the control and AMI group.

\begin{tabular}{|c|c|c|c|}
\hline \multicolumn{2}{|c|}{ Control Group } & & \multicolumn{2}{c|}{ AMI Group } \\
\hline & & 0 & 6 \\
\hline MDA (nmol/g Hb) & $4.76 \pm 1.4$ & $3.99 \pm 1.14^{*}$ & $4.05 \pm 1.35^{*}$ \\
\hline 8-OHdG (10 $\mathrm{dG})$ & $1.25 \pm 0.51$ & $2.52 \pm 1.27 ¥$ & $2.56 \pm 1.36 ¥$ \\
\hline NSSA (U/mg Hb) & $2.44 \pm 0.33$ & $7.80 \pm 2.37 ¥$ & $7.79 \pm 2.65 ¥$ \\
\hline TSSA (U/mg Hb) & $20.78 \pm 4.7$ & $64.90 \pm 18.86 ¥$ & $65.38 \pm 21.49 ¥$ \\
\hline GPX (U/L) & $42.4 \pm 18.3$ & $50.96 \pm 30.60^{*}$ & $57.73 \pm 30.16^{*}$ \\
\hline SOD (U/mg Hb) & $989.6 \pm 156.4$ & $1545.80 \pm 484.5 ¥$ & $1555.30 \pm 518.8 ¥$ \\
\hline GSH (nmol /L) & $39.8 \pm 18.5$ & $41.68 \pm 12.57 *$ & $54.34 \pm 17.67 ¥$ \\
\hline
\end{tabular}

Difference between the control *: $p<0.05 ; ¥: p<0.001 ; \mu$ : $p=0.001$

Difference between 0-6, 0-24, 6-24 were not significant. 
and returned to its baseline levels at the 8th hour. In a study by Kharb S [18], it was shown that GSH levels were lower and MDA levels were higher in AMI patients treated by thrombolytics. Supporting our results, Muzakova V, et al. [19] showed that GPX activity reached its maximum concentration at the $90^{\text {th }}$ minute and returned to its baseline value at the $18^{\text {th }}$ hour after PTCA. In our study we found that SOD levels persisted to be significantly higher at the $24^{\text {th }}$ hour also, GPX and GSH levels without reaching statistical significance were higher in the patients compared to the control group. GPX and GSH increase could be a front line defence against oxidative damage and is rapidly depleted. The down regulation of GPX and rapid elimination of GSH can explain why their concentrations did not reach higher significant levels and elevations in their concentrations could not be detected. In a higher rate usage of tirofiban could be helped to rapid reperfusion at the micro vascular level and it could accelerate the elimination of these front line defences.

\section{Conclusion}

Successful reperfusion provides rapid clearance of free oxygen radicals and supplies oxygen for myocardium under oxidative stress. In contrast to the previous studies, reperfusion obtained by drugs especially with Tirofiban and PTCA during AMI according to the guidelines, affects oxidation-anti-oxidation balance positively and diminishes the oxidative stress and shows advances in reperfusion therapy. The oxidative and anti oxidative parameters (8-OHdG level and SOD activity, etc.) defined in the study can be used to evaluate the success of PTCA, although further large studies are needed.

\section{Limitations of the Study}

The small number of patients in the study is a limitation. Another limitation is that at the time when they were included in the study, the patients were under drug therapy (beta blockers, acetylsalicylic acid, angiotensin converting enzyme inhibitors and statins), which can affect the levels of oxidative and antioxidative stress markers. However, ethically we cannot delay drug administration.

\section{References}

1. Surekha RH, Srikanth BB, Jharna P, Ramachandra RV, Dayasagar RV, et al. (2007) Oxidative stress and total antioxidant status in myocardial infarction. Singapore Med J 48(2): 137-142.

2. Witztum JL (1994) The oxidation hypothesis of atherosclerosis. Lancet 344(8925): 793-795.

3. Bolli R, Patel BS, Jeroudi MO, Lai EK, McCay PB (1988) Demonstration of free radical generation in "stunned" myocardium of intact dogs with the use of the spin trap a-phenyl N-Tert-Butyl Nitrone. J Clin Invest 82(2): 476-485

4. Gur M, Yıldız A, Demirba R, Yılmaz R, Koc A, et al. (2007) The effect of coronary angioplasty on oxidative and anti oxidative status. Arch Turk Soc Cardiol 35(1): 21-27.

5. Beutler E (1971) Red cell metabolism. A manual of biochemical methods. Grune and Strattoa Inc., New York, USA, pp. 112-114.

6. Chiu D, Lubin B (1979) Abnormal vitamin E and glutathione peroxidase levels in sickle cell anemia: evidence for increased susceptibility to lipid peroxidation in vivo. J Lab Clin Med 94: 542-548.

7. Belch JJ, Bridges AB, Scott N (1991) Oxygen free radicals and congestive heart failure. Br Heart J 65: 254-258.

8. Cavalca V, Cighetti G, Bamonti F, Loaldi A, Bortone L, et al. (2001) Oxidative stress and homo cysteine in coronary artery disease. ClinChem 47(5): 887-892.

9. Pucheu S, Coudray C, Vanzetto G, Favier A, Machecourt J, et al. (2005) Assessment of radical activity during the acute phase of myocardial infarction following fibrinolysis: utility of assaying plasma malondialdehyde. Free Radic Biol Med 19(6): 873-881.

10. Miwa S, Toyokuni S, Nishina T, Nomoto T, Hiroyasu M, et al. (2002) Spaciotemporal alteration of 8-hydroxy-2- deoxyguanosine levels in cardiomyocytes after myocardial infarction in rats. Free Radic Res 36(8): 853-858.

11. Nagayoshi Y, Kawano H, Hokamaki J, Miyamoto S, Kojima S (2005) Urinary 8-hydroxy-2-deoxyguanosine levels increase after reperfusion in acute myocardial infarction and may predict subsequent cardiac events. Am J Cardiol 95(4): 514-517.

12. Solen H, Yildiz D, Evin B, Ali V, Tulay A (2009) Oxidative DNA damage and antioxidant defence after reperfusion in acute myocardial infarction. Journal of Investigative Medicine 57(4): 595-599.

13. Toth-Zsamboki E, Horvath E, Vargova K, Pankotai E, Murthy K, et al. (2006) Activation of poly (ADP-ribose) polymerase by myocardial ischemia and coronary reperfusion in human circulating leukocytes. Mol Med 12(9-10): 221-228.

14. Perrelli MG, Pagliaro P, Penna C (2011) Ischemia/reperfusion injury and cardio protective mechanisms: role of mitochondria and reactive oxygen species. World J Cardiol 3(6): 186-200.

15. Dusinovic S, Mijalkovic D, Saicic ZS, Duric J, Zunic Z, et al. (1998) Antioxidative defense in human myocardial reperfusion injury. J Environ Pathol Toxicol Oncol 17(3-4): 281-284.

16. Simic D, Mimic-Oka J, Pljesa M, Milanovic D, Radojevic S, et al. (2003) Time course of erythrocyte antioxidant activity in patients treated by thrombolysis for acute myocardial infarction. Jpn Heart J 44(6): 823-832.

17. Tomado H, Morimoto H, Aoki N (1996) Superoxide dismutase activity as a predictor of myocardial reperfusion and salvage in acute myocardial infarction. Am Heart J 131(5): 849-856.

18. Kharb S (2003) Low blood glutathione levels in acute myocardial infarction. Indian Journal of Medical Sciences 57(8): 335-337.

19. Muzakova V, Kandar R, Vojtisek P, Skalicky J, Cervinkova Z (2000) Selective antioxidant enzymes during ischemia/reperfusion in myocardial infarction. Physiol Res 49(3): 315-332. 